\title{
El horizonte de la atención médica en pediatría: ¿qué hacer en el caso de niños que se encuentran en situación de abandono, conflicto, daño o peligro, aunado a una enfermedad grave?
}

\author{
Jessica H. Guadarrama-Orozco', Guillermo R. Cantú-Quintanilla², Diana Ávila-Montiel', \\ Myriam Altamirano-Bustamante ${ }^{3}$, Ingris Peláez-Ballestas ${ }^{4}$, Cristina Caballero-Velarde ${ }^{2}$, \\ Luis E. Juárez-Villegas ${ }^{1}$, Nahúm de la Vega-Morell ${ }^{5}$, Javier Kelly-García ${ }^{6}$, Adalberto de Hoyos-Bermea ${ }^{7}$, \\ Elisa Dorantes-Acosta ${ }^{1}$, José D. Gamboa-Marrufo ${ }^{1 \dagger}$, Onofre Muñoz-Hernández ${ }^{1,8}$ y \\ Juan Garduño-Espinosa ${ }^{1,8 *}$
}

${ }^{1}$ Hospital Infantil de México Federico Gómez; ${ }^{2}$ Universidad Panamericana; ${ }^{3}$ Instituto Mexicano del Seguro Social (IMSS); ${ }^{4}$ Hospital General de México; ${ }^{5}$ Instituto Panamericano de Alta Dirección de Empresas; ${ }^{6} \mathrm{Hospital}$ de Oncología, Centro Médico Nacional Siglo XXI, IMSS; ${ }^{7}$ Instituto Politécnico Nacional; ${ }^{8}$ Universidad Nacional Autónoma de México. Ciudad de México, México

\begin{abstract}
Resumen
Introducción: Las leyes refieren que los menores no tienen la capacidad para dar su consentimiento informado para su propia atención médica; sin embargo, hay condiciones especiales en las que se les permite determinar lo referente a su salud. Cuanto mayores sean las limitaciones de juicio y experiencia en los menores, menos peso se otorga a los valores y objetivos que expresan; cuanto más adversas sean las consecuencias, se deberá exigir un nivel más alto de autoridad para decidir en nombre del menor, dejando al Estado la capacidad de garantizar el bienestar del menor. Caso clínico: Niña de 12 años con diagnóstico de leucemia linfoblástica aguda LI, con antecedentes familiares y sociales precarios; evolución entorpecida por el desapego al tratamiento y sus condiciones insalubres y pobreza extrema. Ambos padres fallecieron al poco tiempo de iniciar su tratamiento, quedando ella al cuidado de su medio hermana mayor de edad. Se exponen la labor y el dilema ético del oncólogo tratante y del personal del Hospital Infantil de México Federico Gómez en la creación de redes de apoyo con el objetivo de priorizar el bienestar de la menor, sin dar lugar al quebrantamiento y la desintegración familiar, consiguiendo exitosamente su recuperación. Conclusiones: El caso fue sometido al Comité de Bioética Hospitalaria. Se formaron redes de apoyo interinstitucionales para intervenir en la dinámica familiar, resolviendo los requerimientos de la menor, y se consiguió con éxito superar la enfermedad.
\end{abstract}

Palabras clave: Ética médica. Asuntos de bioética. Ética clínica. Comités de ética clínica.

\section{The horizon of medical attention in pediatrics: what to do in the case of children who are in abandonment, conflict, harm or danger situations in combination with a severe disease?}

\section{Abstract}

Background: Laws refer that minors do not have the capability to give informed consent for their own medical attention. However, there are special conditions in which they are allowed to decide about their health. The greater the judgement and

\section{Correspondencia:}

*Juan Garduño Espinosa

E-mail: juan.gardunoe@gmail.com; jgarduno@ himfg.edu.mx
Fecha de recepción: 10-10-2017

Fecha de aceptación: 19-12-2017

DOI: 10.24875/BMHIM.M18000022
Disponible en internet: 14-05-2018 Bol Med Hosp Infant Mex. 2018;75:166-177 www.bmhim.com

1665-1146/C 2018. Hospital Infantil de México Federico Gómez, impreso por Permanyer México SA de CV, todos los derechos reservados. 
experience limitations in minors, the less weight is given to the values and objectives they express. Also, the more adverse consequences might be, the higher the level of authority that is demanded to decide on behalf of the minor, thus granting the State the capability to guarantee the well-being of the minor. Case report: 12-year-old female patient with a diagnosis of acute lymphoblastic leukemia, with precarious social and family background; evolution of the disease obstructed by the disregard of the treatment due to her unsanitary and extreme poverty conditions. Both of her parents died soon after the start of the treatment and she was kept under the care of her half-sister of legal age. The work and the ethical dilemma of the pediatrician and the staff of Hospital Infantil de México Federico Gómez are exposed within the building of support networks with the objective of prioritizing the minor's well-being, without allowing family break-up or disintegration, thus succeeding in her recovery. Conclusions: The case was submitted to the Hospital Bioethics Committee. Inter-institutional support networks were built in order to improve dynamics of the family, thus solving the needs of the minor. Despite the misfortune of the situation, the disease was successfully overcome.

Key words: Bioethics issues. Clinical ethics. Clinical ethics committees. Medical ethics.

\section{Introducción}

En pediatría, se presenta de manera frecuente un gran desafío inherente a la naturaleza de dicha práctica y que se deriva de la imposibilidad de que los niños participen de manera responsable en la toma de decisiones sobre su salud y su bienestar. La gran mayoría de los problemas más escabrosos en pediatría surgen en los niños que son lo suficientemente maduros como para que sea imposible excluirlos por completo del proceso de decisión, pero cuya capacidad para tomar ciertas decisiones importantes es cuestionable.

Las leyes, en casi todo el mundo, refieren que los menores no tienen la capacidad para dar su consentimiento informado y voluntario en su propia atención médica; sin embargo, hay condiciones especiales en las que se permite una reducción de la edad para dar el consentimiento. La justificación habitual de estos casos es permitir a los menores buscar y obtener tratamiento cuando es probable que la exigencia de que los padres den su consentimiento y se les tenga que notificar del caso los desaliente de buscar tratamiento para su propio bienestar. La capacidad de los niños variará conforme al crecimiento y los cambios propios del desarrollo; las diferentes decisiones relacionadas con la atención médica varían en cuanto a las demandas que plantean a la persona que toma las decisiones. Cuando se trata de situaciones complejas, la comprensión y el razonamiento requeridos son mayores que cuando son de menor riesgo y benéficas.

Lo que se discute no es si el menor es capaz de tomar decisiones por sí mismo; más bien, lo que está en duda es su capacidad para entender suficientemente las consecuencias del tratamiento propuesto, de modo que pueda dar o no su consentimiento informado a ese tratamiento. Lo que se busca es que el menor comprenda las repercusiones que estas decisiones tendrán en su vida presente y futura.

Se distinguen tres tipos de facultades para tomar decisiones: a) facultades para la comunicación y la comprensión de la información; b) facultades para el razonamiento; y c) deliberación y facultad de aplicar un conjunto de valores o una concepción del bien. Los niños menores de 12 años son más propensos que los niños mayores o los adultos a ver el locus de control como algo externo a ellos. Lewis ${ }^{1}$ descubrió que los niños de entre 6 y 9 años con frecuencia no se percibían como los que tomaban la decisión, ni siquiera cuando lo estaban haciendo. Este punto cobra relevancia en lo concerniente a la voluntariedad; si los menores ven el locus de control como externo a ellos, creerán que la elección no es algo que esté en sus manos y no opondrán resistencia a que los demás impongan sus propias elecciones. Entre los 7 y 13 años, ya han dejado el pensamiento mágico, pero aún tienen problemas para anticipar el futuro?

Los niños aprenden que deben obedecer y esto condiciona que los menores de 14 años logren hacer frente a las figuras de autoridad (padres, médicos, maestros).

Quizá lo más importante para el razonamiento sean varias capacidades que Piaget llamó la etapa de las operaciones formales en el desarrollo cognitivo ${ }^{3}$. Alrededor de los 5 años, los niños empiezan a explicar con puntos vista bastante mágicos la causalidad de las enfermedades; no es sino hasta los 12 o 13 años cuando la mayoría de ellos empieza a entender «que hay múltiples causas de enfermedad, que el cuerpo puede responder de forma variable y que factores huéspedes dentro del cuerpo interactúan con el agente para causar y curar la enfermedad". De manera general, las pruebas de desarrollo sustentan la conclusión de que los menores, a partir de los 14 o 15 años en general, 
ya han desarrollado las diversas facultades necesarias para ser capaces en la toma de decisiones que atañen a su atención médica en un nivel más o menos comparable al alcanzado por la mayoría de los adultos.

También existe lo que se han llamado reglas subjetivas, que son de dos tipos: 1) menor condicional, cuando un menor puede dar su consentimiento si su vida corre peligro; y 2) menor maduro, que puede dar su consentimiento si entiende la naturaleza y los propósitos del tratamiento propuesto ${ }^{4}$.

La regla del menor maduro conserva la presunción de que los menores no tienen la capacidad para dar su consentimiento a su propio tratamiento; exige que para rechazar esa presunción, se demuestre su «madurez», esto es, la comprensión de la naturaleza y los objetivos del tratamiento propuesto en todos los casos. Esta regla ha sido restringida en casi todos los casos a aquellos niños que están muy cerca de la mayoría de edad, normalmente nunca menos de 14 años, con independencia de las facultades generales del menor para decidir.

Las leyes occidentales afirman que toda persona, desde que nace y hasta que muere, cuenta con capacidad de goce, considerada como la aptitud para ser titular de derechos o sujeto de obligaciones. Esto implica que, si bien se puede carecer de capacidad de ejercicio, no por ello se carece de personalidad jurídica. Por lo tanto, se entiende que los derechos de los niños estén tutelados por la ley hasta que el individuo alcance una capacidad plena para el ejercicio ${ }^{5}$.

Sin embargo, esta capacidad no es cuestión de grados: es un concepto de umbral; y entonces, ¿dónde o en qué nivel de las aptitudes para la toma de decisiones y la ejecución de una decisión particular se debe situar este umbral, de modo que separe a los capaces de los incapaces? En el caso de los niños, dada la presunción legal de incapacidad, nos preguntaremos qué tan buenas deben ser sus aptitudes y su desempeño para tomar decisiones en lo que atañe a una decisión sobre su salud como para que rechace la presunción de su incapacidad y para que sean declarados capaces de decidir por sí mismos, y cómo establecer el nivel de aptitud necesaria para ser capaz en la toma de decisiones de una manera no arbitraria (fijar solo la edad cronológica como tope). La respuesta a estas cuestiones es que el nivel apropiado será un equilibrio de los principales valores en juego, que son la autodeterminación y el bienestar del paciente.

Ahora bien, en los niños se reconoce un tercer valor sustancial: el interés de los padres por tomar decisiones importantes acerca del bienestar de sus hijos menores. Los padres, que se preocupan por el bienestar de sus hijos y los conocen a ellos y sus necesidades mejor que nadie, serán capaces de garantizar que las decisiones tomadas son por el bienestar de sus hijos más que cualquier otra persona. Esto no quiere decir que los padres puedan imponer su elección cuando esta no sea la mejor para sus hijos, porque no son sus dueños, sino que son sus tutelares. En segundo plano, son los padres quienes afrontarán las consecuencias de las elecciones en los tratamientos de sus hijos; por lo tanto, parece contradictorio forzarlos a asumir las consecuencias del tratamiento elegido y negarles la posibilidad de elegir. Sin embargo, es el niño quien asume las consecuencias principales de la elección, y por ello es el bienestar del niño lo que debe determinar principalmente la elección.

Los hijos no son propiedad de los padres y no pueden hacer con ellos lo que deseen; los niños son tablas rasas, y solo a través de un proceso de socialización y desarrollo van a adquirir valores, metas y criterios, junto con la suficiente experiencia y las facultades para que justifiquen el respeto a sus decisiones.

Cuanto menor sea un niño, menos peso daremos a las preferencias que expresa y a sus puntos de vista sobre su propio bien; cuanto mayores sean las limitaciones de juicio y experiencia en los niños y adolescentes, menos peso se conferirá a los valores y objetivos que expresan.

Las decisiones por sustitución corresponden esencialmente a los padres, porque la familia, como dice el Dr. Diego Gracia, es desde su raíz una institución que tiene el derecho a dotar de contenido a la beneficencia del niño, siempre y cuando no se traspase el límite de la no maleficencia. Vigilar esto último es función absoluta del Estado ${ }^{6}$, de lo cual se desprende que la autoridad paterna no es absoluta, y cuando el bienestar y la vida de los hijos se encuentran en peligro, el Estado tendrá la obligación de intervenir.

La familia es una institución social valiosa, es el contexto en que se desenvuelven las propias facultades de los niños para la vida íntima y social. Por esto, la familia debe sentirse libre de cualquier control o intrusión ajenos; un aspecto de esta libertad es el derecho de tomar decisiones importantes sobre el bienestar de sus miembros aún incapaces.

A diferencia de los adultos, el bienestar de los niños depende en menor medida de sus preferencias personales del momento, y en mayor medida de las condiciones objetivas necesarias para fomentar su desarrollo y oportunidades en el futuro. Los padres son la autoridad suprema en la determinación del bien del 
niño. El involucrar a los niños en decisiones acerca de su tratamiento promueve su bienestar, ya que aumenta su disposición a cooperar con el médico y su familia, lo cual mejora la evolución de la enfermedad.

México suscribió la convención sobre los Derechos del Niño el 19 de junio de 1990, cuya observancia es obligatoria por expresa disposición del artículo 133 de la Constitución General de la República Mexicana, de cuyos artículos 2, 3, 9, 12, 19, 20, 21 y 27 se deriva que los Estados parte tomarán todas las medidas apropiadas para garantizar «que el niño se vea protegido". Además, en dicha Convención se declara que «el interés del niño resulta un principio rector en quienes tienen la responsabilidad de su educación, salud y alimentación » ${ }^{7}$, pero el principio de mejor interés aplica a aquellos sujetos menores de edad o incapaces de manera temporal que tienen el potencial de consideración moral futura y son capaces de desarrollar facultades para llegar a ser adultos con autodeterminación.

Por otra parte, excepto cuando los intereses más básicos de los niños están en juego, un mero fallo por parte de los padres en su intento por optimizar el interés del niño no será suficiente para desencadenar la intervención justificada de un tercero.

Entonces, el principio del mejor interés solo ha de servir como un ideal regulativo, un principio orientador, no como un requisito estricto, en situaciones en las que no estén en riesgos los intereses básicos y primordiales de un niño (alimentación, salud, educación), ya que los propios intereses legítimos de los padres pueden entrar en conflicto con hacer aquello que maximiza el bienestar del niño, y a veces pueden tomar prioridad sobre ellos. Se espera, en realidad, que los padres hagan sacrificios por sus hijos, pero eso no los obliga a frustrar sus propios intereses más importantes, cuando hacer esto pudiera lograr un aumento adicional no indispensable de beneficio para su hijo.

Ferdinand Shoeman ${ }^{8}$ explica lo poco factible que es que el principio del mejor interés guíe estrictamente la decisión de los padres sobre sus hijos menores: "se debe considerar que la familia es un arreglo íntimo con sus propias metas y propósitos [...] por tanto, no sería adecuado imponerle a ese arreglo [...] principios liberales abstractos». No se debe entender que el principio del mejor interés, como principio orientador para las decisiones de los padres, exige la optimización literal del interés del hijo en todos los casos, sino que además los principios de intervención adecuados permitirán a los padres un amplio margen de flexibilidad; es decir, tolerar alejamientos o distanciamientos admisibles de lo que pudiera ser lo mejor para el hijo a fin de proteger a la familia de intrusiones que vulnerarían la privacidad que requiere para poder desarrollarse como una unión íntima, cuyo valor para los que participan en ella dependen de esa intimidad. Por ejemplo, elegir una religión diferente de la que rige en la sociedad (p. ej., la santería), mientras no dañe al menor y a la sociedad, no se le podría impedir a la familia.

¿Pero cuál es el umbral para la intervención del Estado en el cuidado de los hijos? ¿Cuál es el momento justo en que el Estado puede intervenir con los padres en la tutela del menor? ¿Cuándo es preferible que transcurra la infancia del menor en albergues y casas-hogares, en lugar de hacerlo en el seno familiar? La variable más importante tendrá que ver con las consecuencias especiales de la elección de los padres para el bienestar del niño, las cuales pueden oscilar entre claras y sustancialmente benéficas (p. ej., evitar la incapacidad grave e irreversible o la pérdida de la salud de forma permanente, la educación básica, creencias y religión), pasando por otras triviales o insignificantes, hasta llegar a situaciones seriamente dañinas (muerte). Cuanto más adversas sean las consecuencias esperadas para el niño, más razonable será exigir un nivel más alto de intervención de terceros cuando las decisiones de los padres no tengan un nivel apropiado de capacidad de elección en favor del interés del menor. A mayor adversidad de las consecuencias se deberá exigir un nivel más alto de capacidad para decidir en nombre del menor, la posibilidad de intervenir a la familia y que el Estado garantice el bienestar del menor.

En este artículo se presenta el caso de una paciente cuya pediatra oncóloga tratante y el equipo sanitario del Hospital Infantil de México Federico Gómez (HIMFG) se enfrentaron al dilema de intervenir jurídicamente a la familia en pro del bienestar máximo de la menor, versus vigilar y participar estrechamente e intervenir socialmente, sin desmembrar a la «familia» en un contexto circunstancial verdaderamente adverso. Exponemos cómo la intervención puede darse desde redes de apoyo paralelas a la adversidad que sustenten a la familia, con el objetivo de priorizar el mejor interés del menor, sin dar lugar al quebrantamiento y la desintegración familiar, aun cuando la situación familiar pueda ser calificada de índole catastrófica, al margen de la intervención jurídica.

\section{Caso clínico}

A mediados de 2008, la paciente de 9 años fue llevada por su familia al HIMFG, referida desde el hospital 
pediátrico de segundo nivel con diagnóstico de síndrome mieloproliferativo.

Se registran los siguientes antecedentes sociales de la niña (Figs. 1 y 2): madre de 45 años, analfabeta y aparentemente sana; padre de 39 años, analfabeto, ayudante de albañil, alcohólico desde los 8 años, quien, a referir de la madre, ejerce violencia intrafamiliar. Tiene seis hermanos; uno falleció a los 7 meses de vida (se desconoce la causa). La familia reside en el Estado de México en una casa rentada, en hacinamiento y zoonosis, ubicada cerca de un paso de aguas negras, dentro de los límites de un tiradero de basura municipal, que cuenta con los servicios básicos (agua y luz). La paciente fue un producto a término sin control prenatal, atendida en casa por el padre, como todos sus hermanos. Cuenta con esquema de inmunizaciones incompleto. Alimentación a base de seno materno exclusivo hasta los 11 meses. Desarrollo psicomotor adecuado, no escolarizada. La madre refiere hospitalización por quemaduras en los miembros inferiores a los 3 años de edad con agua caliente que utilizaban para bañarse.

A su ingreso se diagnostica con síndrome anémico, síndrome infiltrativo, síndrome febril, síndrome mieloproliferativo y desnutrición crónica descompensada. Durante su estancia se establece el diagnóstico de leucemia linfoblástica aguda $\mathrm{LI}$, de alto riesgo por leucocitosis, y se inicia tratamiento. Se documenta remisión al día 21 de iniciar el tratamiento en su fase de inducción. Un mes después se alerta a la madre sobre el atraso en la administración planeada de la quimioterapia. Durante los siguientes meses, la paciente recibe tratamiento de quimioterapia intratecal (intensificación y mantenimiento) de manera intermitente, cancelada en dos ocasiones por la aparición de complicaciones infecciosas leves respiratorias. Se realiza aspirado de médula ósea para control y se tratan las dermatosis reincidentes atribuibles a la mala higiene.

En octubre 2008, la niña acude acompañada de una hermana mayor, de 16 años, quien desconoce el tratamiento de la paciente, por lo que se solicita apoyo al Departamento de Trabajo Social, quien solicita a los padres acudir para explicarles el tratamiento, y ellos acuden. A principios de enero de 2009 termina la etapa de reinducción y la paciente acude a sus citas en forma intermitente, debido a lo cual la administración de la quimioterapia es irregular, por lo que se insiste en el apego al tratamiento (Fig. 1).

En junio de 2009, como parte de la actividad de rescate de pacientes, se hace visita domiciliaria por parte de la trabajadora social, a partir de la cual se reporta que la paciente pertenece a una familia extensa, que incluye a otros menores de edad y adultos, vive en terrenos baldíos donde se asienta el tiradero municipal de basura a cielo abierto; habitan un jacal construido con desperdicios industriales, bajo condiciones de hacinamiento, promiscuidad, alcoholismo, pobreza extrema, insalubridad y zoonosis, y no cuentan con servicios básicos en el domicilio. Quienes realizan la visita encuentran a la madre en mal estado de salud, postrada en cama, incapaz de cuidar de sí misma y de sus hijos. Una hija de 12 años es quien cuida y atiende a la madre, a la paciente, a dos hermanas menores y a una sobrina. Se entrevista al padre, quien se observa desaseado y bajo los efectos del alcohol; se le cita en el HIMFG para ese mismo mes. La paciente acude al hospital acompañada de su padre. Se realiza interconsulta con psicología, en la que se reporta que el padre presenta un coeficiente intelectual limítrofe y no mantiene buen vínculo con la paciente, además de que no se identifica compromiso con la salud de su hija, pero se reporta a la niña de 10 años, consciente y al tanto del tratamiento y de las consecuencias futuras de no recibirlo adecuadamente. Se insta al padre acerca de la adherencia al tratamiento y se le enfatiza que debe asumir la responsabilidad de este. El médico tratante acuerda con la familia que la paciente acudirá al hospital cada lunes, con la finalidad de generar un hábito que les facilite acudir a sus citas.

En las siguientes consultas, la paciente acudió acompañada de dos hermanos, quienes desconocen el tratamiento previo o el estado del mismo, e informan de que ha fallecido la madre (se desconoce la causa). Se informa al Sistema Nacional para el Desarrollo Integral de la Familia (DIF) para conseguir un acercamiento más cercano con la familia y evitar el abandono del tratamiento.

En julio de 2009 se notifica la situación a la Procuraduría de Defensa del Menor y de la Familia. Una de sus hermanas (a pesar de ser menor de edad), se compromete a participar en el tratamiento de la paciente.

A finales de año se sometió el caso al Comité de Bioética Hospitalaria, que recomendó que el Departamento de Trabajo Social y el Departamento Jurídico de la Institución se coordinaran con las oficinas del DIF a fin de contactar al padre para insistir en responsabilizarlo del tratamiento. Se conoce que la madre tiene dos hijas de una pareja previa: una de 21 años, quien vive con su pareja y tiene una hija de 1 año en el hogar de la familia de la paciente, y otra hermana de 17 años. 


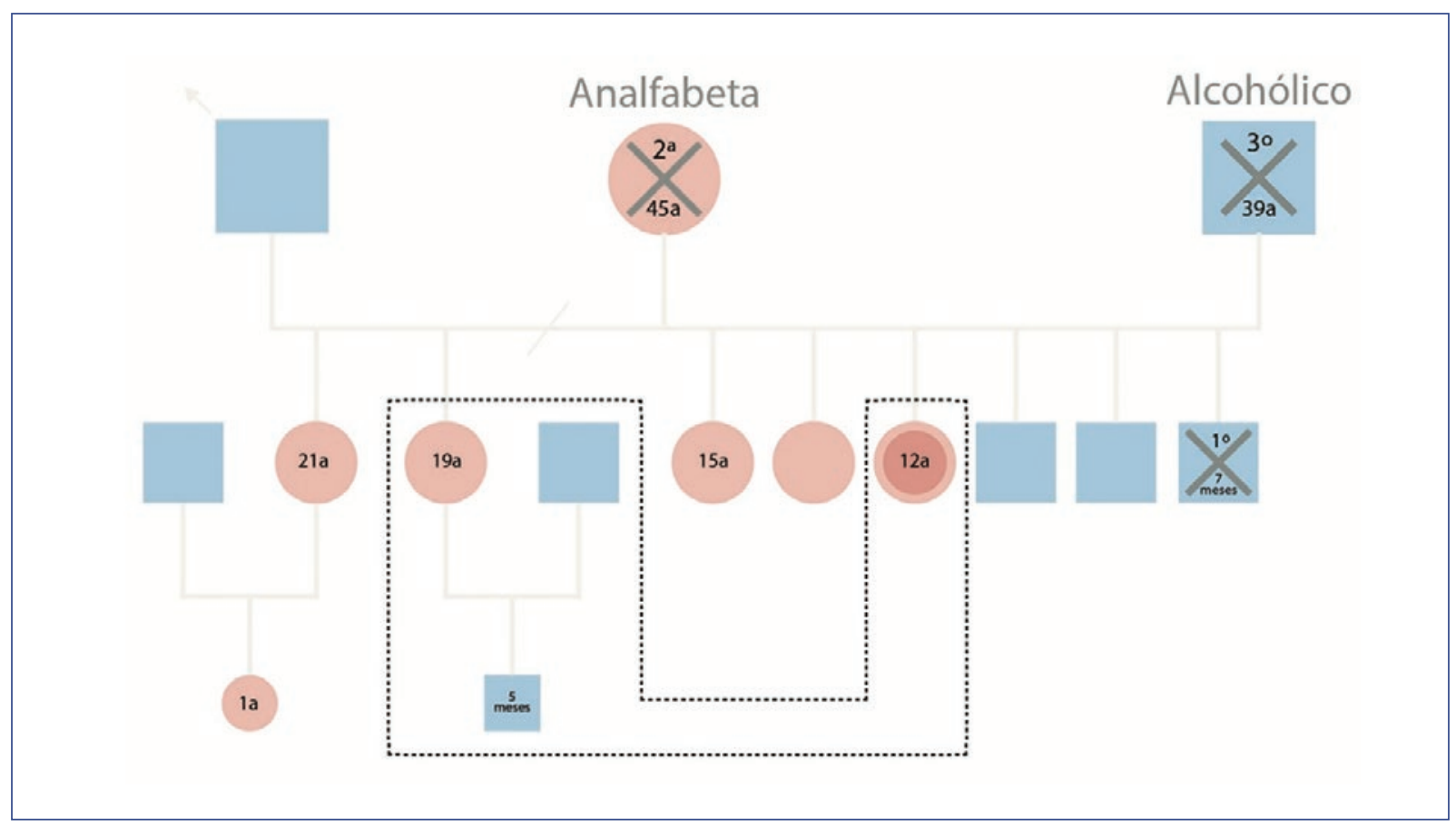

Figura 1. Familiograma de la paciente.

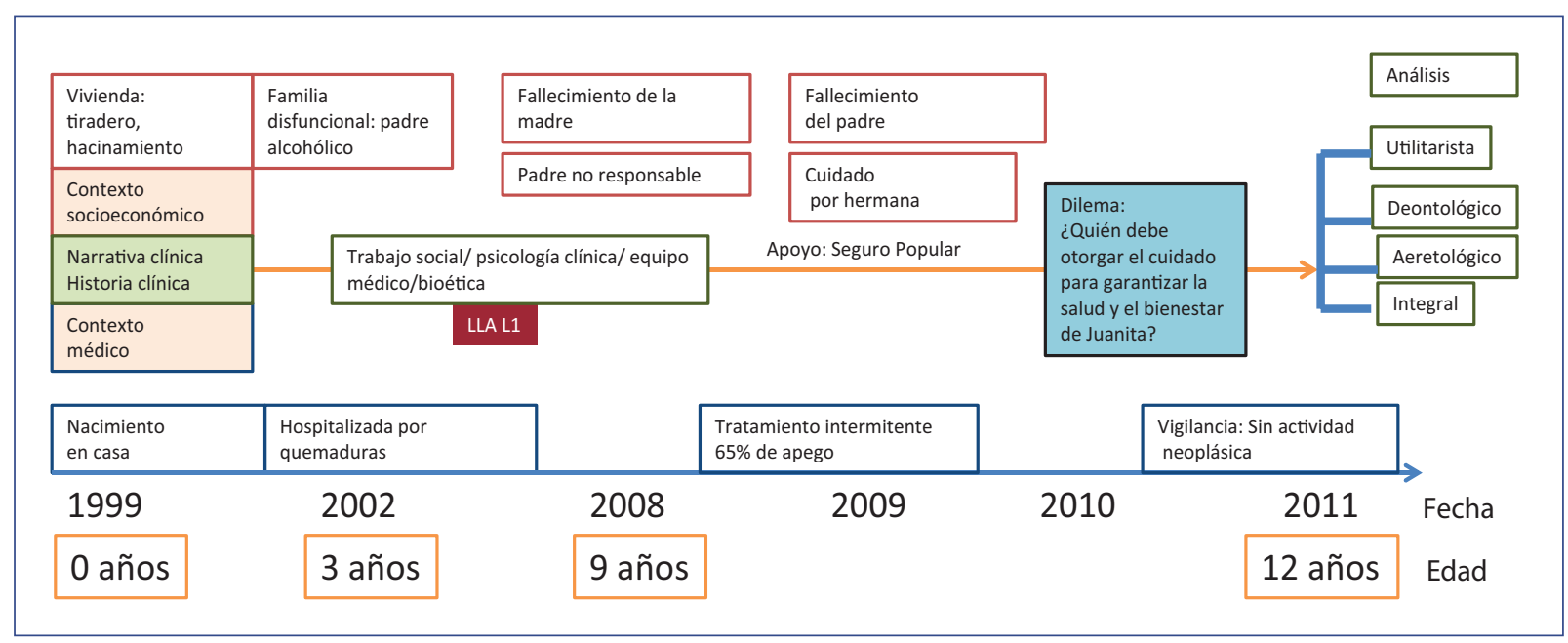

Figura 2. Narrativa clínica y alternativas bioéticas.

Después del fallecimiento de la madre no existen recursos económicos ni redes de apoyo para lograr el apego al tratamiento; asimismo, no se identifica a nadie en la familia que se responsabilice por el cuidado de los menores. En diciembre de 2009, el DIF realiza una visita y asigna cita para acudir al HIMFG para la aplicación del tratamiento intratecal. En febrero de 2010, la paciente asiste para la aplicación de la quimioterapia de mantenimiento correspondiente a la semana 61 (desde su diagnóstico), aunque en la práctica correspondió a la semana 36 por aplicación.

En abril de 2010, después de un tratamiento irregular, acude acompañada de su medio hermana mayor, de 18 años, ya que el padre también ha fallecido. En julio de ese mismo año, posterior al estudio social del DIF, la medio hermana de 18 años acepta fungir como tutora legal de la paciente. La niña se traslada a su nuevo domicilio, donde comparte vivienda con su medio 
hermana, su pareja y su hijo de 5 meses, siendo la pareja de su hermana quien se encarga de solventar los gastos de la manutención. Los demás hermanos quedaron bajo el cuidado de su otra medio hermana de 21 años. El mayor deseo de la paciente siempre fue seguir en su casa acompañada de sus hermanos menores, independientemente de la situación social y sanitaria a la que se enfrentaba. Ella era capaz de respaldar con razones claras y fundamentadas su deseo de permanecer en casa.

En diciembre de 2010, durante su hospitalización para tratamiento, se mantuvo sin familiar acompañante. En noviembre de 2011, a los 12 años y 10 meses de edad, y con un tratamiento de 120 semanas, se deja en vigilancia con evidencia de remisión.

\section{Análisis}

A fin de abordar el dilema, se expone a continuación un conjunto de preguntas que permiten, en primer término, especificarlo y caracterizar el contexto en que ocurre. Además, se incorporan dos enfoques conceptuales con su respectivo marco de referencia ético, que pueden auxiliar en el análisis del dilema.

\section{Discernimiento ético por el método integral}

- Contexto en el que ocurre el dilema y cuál es el hecho: paciente femenino de 9 años, con leucemia aguda linfoblástica de alto riesgo. La paciente forma parte de una familia en pobreza extrema; acude a sus consultas en malas condiciones de higiene y con diferentes cuidadores. Desde el inicio del tratamiento no hay un cumplimiento apropiado; solo se obtuvo el $64 \%$ del plan terapéutico proyectado. La familia de la paciente está desintegrada, los padres fallecen durante el proceso de tratamiento y los hermanos intentan participar, con sus recursos limitados, en el cuidado de la paciente.

- ¿Cuál es el bien que se busca? El bien mayor es la protección de la vida de la paciente en sus dimensiones biológica, psicológica y social.

- ¿Quiénes son los beneficiarios del bien? La paciente y su familia.

- ¿Quiénes son los actores principales? La paciente, su familia y el personal de salud del servicio de oncología.

- ¿Cuáles son las circunstancias, los costos y los riesgos? Se trata de una paciente con leucemia aguda linfoblástica de alto riesgo. La familia entiende de manera limitada el diagnóstico, el tratamiento y sus implicaciones. La paciente proviene de una familia disfuncional, cuyo principal detonante es la pobreza y el alcoholismo que aceleran la enfermedad y la muerte de ambos padres, dejando huérfanos a los cuatro hermanos menores de edad. El único apoyo posible es el de las dos medio hermanas que son mayores de edad.

- ¿Cuál es el dilema? El dilema ético que se plantea al personal de salud es solicitar que la patria potestad de la menor se retire temporalmente de la familia y se la envíe a una casa-hogar hasta que se supere la enfermedad, o bien que la paciente continúe viviendo en las condiciones actuales y formar redes de apoyo y de vigilancia lo suficientemente fortalecidas para garantizar que pueda superar la enfermedad. La razón fundamental para considerar el apartar a la paciente de su núcleo familiar radica en que se observa una falta de adherencia al tratamiento que pone en riesgo la vida de la menor. La paciente (cuya experiencia en la toma de decisiones importantes es aún inmadura), desde la primera consulta, expresa de forma explícita razonable el deseo de permanecer junto a sus padres y hermanos.

\section{Estrategia analítica para resolver el dilema}

\section{Perspectiva utilitarista}

El mayor beneficio o bienestar que desencadena la intervención de terceros, en este caso, es el riesgo de no poder recuperar la salud y garantizar la supervivencia de la paciente. Por ello, el motivo de intervención de ninguna manera es trivial. Desde el punto de vista del personal de salud involucrado, el beneficio esperado de retirar la tutela de los padres de forma temporal o definitiva superará las cargas impuestas, tanto a la paciente como a su familia. Si se considera, además, el potencial costo-beneficio, el envío de la paciente a una casa-hogar sería lo más recomendable, ya que podría terminar exitosamente el tratamiento y tener adicionalmente los cuidados necesarios. Sin embargo, ¿qué tanto garantiza una casa-hogar en México el cuidado de la paciente?, ¿no es mejor fortalecer a la familia y responsabilizarla del cuidado de sus integrantes?

\section{Perspectiva deontológica}

El deber del equipo médico es no dañar al paciente y siempre que sea posible beneficiarlo a través de la recuperación de la salud, así como garantizar su supervivencia cumpliendo con el deber de buscar las 
condiciones que garanticen su supervivencia. Este propósito se ve reforzado por el hecho de que interviene un equipo interdisciplinario en el tratamiento, que incluye las dimensiones biológica, psicológica y social. Es deber del médico responsable y del equipo que colabora en el cuidado del paciente proyectar las necesidades que surjan a partir del diagnóstico, así como los cambios y arreglos que sufrirá la familia para que su integrante enfermo pueda superar la adversidad, valorar si la familia será capaz de afrontar este reto o si requerirá ayuda externa en distintas aristas como puntos de fragilidad para sobrellevarlo, o si los considera totalmente incapaces de afrontarlo.

Por otra parte, la paciente, con 9 años, debe ser consultada sobre su deseo de continuar viviendo con su familia $o$ ir a una casa-hogar (asentimiento informado), explicándole los pros y contras de su decisión; sin embargo, su opinión en este momento será solo una opinión, pues no se puede ponderar esta como autodeterminación debido la falta de experiencia y proyección futura que a los 9 años puede un menor tener.

A lo largo de la enfermedad, la paciente ha tenido que afrontar la muerte de ambos padres y la falta de recursos, tanto sociales como emocionales, para enfrentar su enfermedad, lo cual a los 10 años le hizo adquirir mayor experiencia y facultades para poder opinar y ser parte activa ella misma de las decisiones sobre su salud, si se le permite y ella lo desea.

\section{Perspectiva aretológica}

La pregunta esencial es: ¿estoy evitando el mal? Algo es bueno si perfecciona, si busca alcanzar la plenitud en la naturaleza inherente de quien actúa. El tratamiento oportuno también puede contribuir a que la paciente alcance su mejor desarrollo; para el personal de salud también habrá un perfeccionamiento, puesto que se cumple con su vocación al servicio de la vida. Se da prioridad a la sindéresis, la integridad, la proporcionalidad y la virtud. Desde esta perspectiva ética se tendría que construir una red social para apoyar a la paciente y su familia, de manera integral, que garantice la adherencia al tratamiento y los cambios en el estilo de vida que sean efectivos. El mayor bien para la paciente sería permanecer en su núcleo familiar en las mejores condiciones sanitarias posibles y de alimentación, las cuales se pueden lograr a través de la construcción de una red de apoyo a la familia. Esta perspectiva tiene como limitante la posibilidad realista y factible de llevar a cabo tales cambios, mismos que requieren procesos que generen cambios desde las raíces fundamentales de esta familia, lo que conlleva tiempo y la inversión de múltiples esfuerzos.

\section{Análisis integral}

Dado que, desde el punto de vista aretológico y deontológico, la decisión más adecuada sería mantener a la paciente en su núcleo familiar, lo que tendría que promover el personal de salud a través de sus redes de apoyo sería un mejoramiento de la calidad de vida de la paciente y sus hermanos (también menores de edad). En el marco de su contexto social, es un reto para el personal sanitario coordinar los esfuerzos sociales para que la paciente sobreviva a la enfermedad, pero que además ella y sus hermanos tengan acceso a educación, vivienda saludable y alimentación segura. Recordemos que la capacidad de un adolescente puede variar con el tiempo de acuerdo con los cambios en su condición, de modo que puede ser intermitente o fluctuante, pero este es otro punto a abordar: la capacidad de la paciente de ser partícipe en la toma de decisiones y que los médicos responsables y demás partícipes valoren esta intervención (Fig. 1).

\section{Discernimiento ético de acuerdo con Peter Kemp}

Desde el marco general de la ética narrativa, este acercamiento nos permite entender la dignidad humana como una cualidad que forma parte de la integridad psíquica y corporal del ser humano ${ }^{9}$. En este enfoque se aplican cuatro principios que pueden contribuir a ordenar la discusión de los casos que se analizan.

\section{PRINCIPIO DE DIGNIDAD}

En primer lugar, este principio afirma que cada sujeto vale por sí mismo, que no tiene precio, por lo que se le debe respetar y proteger. La dignidad no está en función del lugar donde se nace o se vive, no depende de quiénes son los padres, del nivel social o económico, ni de la educación o la historia personal. En el caso que se aborda, el Servicio de Oncología del HIMFG organizó un equipo de trabajo con Trabajo Social, Psicología, DIF y Departamento Jurídico para atender y recuperar la salud, y lograr mantenerla aun en medio de la indigencia, la ignorancia y la miseria en que viven la paciente y sus hermanos. Es de acuerdo con este principio que los médicos deben enfocar su esfuerzo en rescatar ese valor intrínseco de la paciente, sin impedir que tome parte de las decisiones respecto a su vida aduciendo 
su edad y falta de experiencia, valores y destrezas como pretexto obvio para anular sus opiniones.

\section{PRINCIPIO DE INTEGRIDAD}

En segundo lugar, el principio de integridad hace referencia a lo que no se debe alterar o dañar, que tiene una totalidad, una coherencia, que le da unidad de sentido y vida. Desde esta perspectiva, el Servicio de Oncología busca y consigue sortear las dificultades de la realidad socioeconómica de la paciente para darle un tratamiento largo y costoso. En este caso, dar un tratamiento enfocado exclusivamente a curar el cáncer implicará no dar una atención integral a la paciente, así como una falta de visión para anticipar el futuro sin prever fracasos posteriores secundarios a no abordar integralmente el caso clínico.

\section{Principio de VUlnerabilidad}

Finalmente, en relación al principio de vulnerabilidad en sus tres dimensiones, tenemos: a) en lo biológico y corporal, dada la fragilidad de la vida orgánica humana, se intenta establecer un diagnóstico y un tratamiento manteniendo las condiciones de continuidad espacio-temporal y de higiene y alimentación mínimas, a fin de evitar complicaciones posteriores; b) en lo social, la intención es conservar un ser humano con una coherencia narrativa, que reciba los bienes y servicios necesarios para conservar la vida, con el propósito de rehacer su historia desde la pobreza y el abandono, hasta el cuidado y la protección; y c) en lo cultural, dada la fragilidad de la tradición de unos valores establecidos localmente, el enfoque es a partir de encontrar y dar un sentido a la vida contando con su hermana como persona responsable.

\section{Principio de AUtonomía}

En este caso se encuentra disminuido a causa de la falta de experiencia de la paciente en la toma de decisiones, debido a su edad y un paternalismo justificado por parte del HIMFG. Este principio se reconduce a través del principio de subsidiariedad aplicado por el equipo del HIMFG, para que entre en acción el trabajo en equipo de los diferentes actores que son parte de la atención de la paciente, para sacarla adelante, aun a pesar de un entorno hostil. Dada la presunción legal de incapacidad, la pregunta a plantearnos es: ¿qué tan buenas deben ser sus aptitudes y su desempeño para tomar decisiones en lo que atañe a una decisión particular y poder ejercer su autonomía, como para aprobar o rechazar la presunción de incapacidad? El bienestar de los niños, en este caso de la paciente, dependerá más de las condiciones objetivas necesarias para fomentar su desarrollo y oportunidades que de sus preferencias individuales del momento. Por ello, para rechazar dicha presunción se deberá «demostrar su madurez», esto es, su comprensión de la naturaleza y los objetivos del tratamiento propuesto, que han sido comprobadas.

\section{Discusión}

En el horizonte entre la ética del mínimo exigible y la ética del máximo deseable se concibe la bioética con un fuerte componente aspiracional, una búsqueda continua de lo mejor que pueden ofrecer los agentes de la salud, integrados en un equipo interdisciplinario, para cada paciente a ellos encomendado. La bioética consigue así adaptarse a los cambios científicos y tecnológicos en constante transformación por la colaboración de innumerables equipos de trabajo, con el anhelo de la perfección y la virtud. Con ese marco de referencia, con unos principios de reflexión y unos elementos de juicio o criterios de discernimiento, se elaboran planes de acción para decidir qué es lo más conveniente para el paciente y su familia, lo que permite abordar los dilemas éticos que se presentan en el ejercicio de la profesión.

¿Pero qué es un dilema ético? Para Prilleltensky, et al., son los problemas morales encontrados en la práctica profesional y que provocan graves conflictos internos, ya que implican acciones que confrontan valores y que con frecuencia enfrentan tanto a colegas como a empleados 0 a diferentes sectores del entramado social ${ }^{10}$. Estos dilemas se han clasificado en conflictos relativos a los valores personales, conflictos con otros profesionales, conflictos con los pacientes $y$, por último, conflictos con las organizaciones.

No es infrecuente encontrar en la práctica pediátrica (más que en otras especialidades) situaciones en que la actuación de los padres se juzga por el personal sanitario como insuficiente o inapropiada en relación con sus hijos enfermos. El médico, como responsable del niño en el proceso de enfermedad, requiere en ocasiones ejercer un juicio no intervencionista sobre el comportamiento de los padres en torno al menor, y dárselo a conocer para promover mejoras; sin embargo, de no observarse cambios, debe hacer partícipes a otros miembros de la institución y extrainstitucionales 
(si el caso lo amerita) para, en equipo, intervenir a la familia en pro del bienestar del menor.

La Fiscalía Central de Investigación para Menores es la instancia facultada para intervenir en los casos en que se encuentre un niño en situación de daño, riesgo o peligro, y que se requiera para su protección su canalización a un albergue temporal o institución pública o privada de asistencia social. La Fiscalía tiene como objetivo salvaguardar en todo momento el interés superior del menor, manteniendo su derecho de estar al cuidado de sus progenitores; cuando esto no fuera posible, debido a que los padres fueran los probables responsables de una conducta contra la integridad de los menores, o bien porque no se les hubiese podido ubicar, deberá proceder de conformidad con lo establecido en los artículos 303 y 305 del Código Civil para el Distrito Federal, respecto al orden en que los familiares tienen la obligación de cuidado y alimentos hacia los menores.

Solo en caso de que no sea posible la entrega del niño o niña con sus padres o familiares, de conformidad con las fracciones anteriores, la Fiscalía Central de Investigación para Menores deberá, bajo su estricta responsabilidad, canalizarlo para cuidados y atenciones al Albergue Temporal de la Procuraduría o a la institución pública o privada de asistencia social que corresponda, de conformidad con lo siguiente: tratándose de niños o niñas de 0 a 12 años, y siempre que exista la disponibilidad física, serán canalizados al Albergue Temporal de la Procuraduría General de Justicia del Distrito Federal.

No debemos olvidar que, en México, la situación para los niños que son referidos a una institución de este carácter es compleja, porque no solo están huérfanos de padres, sino en ocasiones también del Estado, pues México ocupa el segundo lugar en América Latina en frecuencia de niños abandonados. El Estado puede ser omiso en cumplir con su obligación de regular y supervisar la operación de centros públicos y privados donde se ha dejado a miles de niños bajo su cuidado, debido a la sobrepoblación de estos. Desde hace 30 años se han enviado niños a albergues sostenidos por organizaciones de la sociedad civil ante la incapacidad del Estado y de la sociedad para hacer frente a una problemática en aumento, una niñez que con frecuencia es víctima de orfandad, maltrato y abusos $^{7,11}$.

El umbral, entonces, para la intervención del Estado deberá ser el daño al menor; es decir, cuando se identifica «maleficencia». Sin embargo, ello no implica el retiro obligado de la custodia o tutela del menor, porque antes debe intentarse el fortalecimiento de la familia a través de intervenciones de mediana y alta complejidad, cuando el caso lo permita. «La directriz fundamental es la prioridad del bien del menor sobre cualquier otro interés y no puede tener una rígida e inflexible definición»; este es el principio rector y orientador que guía tanto a las autoridades como a la sociedad para adoptar las medidas necesarias para hacer respetar los derechos fundamentales de los menores, teniendo en cuenta siempre que cada niño, en cada conflicto, «merecerá una solución específica y distinta según cada situación» ${ }^{12}$.

Los padres son quienes influyen en las decisiones sobre el tipo de acciones que podrían beneficiar más al hijo, y con frecuencia estas decisiones tienen un impacto más allá del presente; es por ello por lo que los médicos tienen la responsabilidad de formular un juicio independiente sobre el mejor interés para el paciente, así como de evaluar y cuestionar las decisiones de los padres con proyección futura.

En el caso que nos ocupa fue evidente la falta de atención indispensable ante la gravedad de la enfermedad que presentaba la paciente. La familia sobrevivía bajo condiciones de pobreza extrema y abandono; sin embargo, la enfermedad de la niña fue el detonante para que hubiese intervención en la situación de esta familia. De no haber sido por la enfermedad, ¿todos los menores habrían seguido en el mismo estado de abandono? No se puede culpar a los padres de abandono o negligencia en el cuidado, pues dentro de la medida de sus posibilidades intelectuales, sociales y económicas hacían lo posible por dar cumplimiento al tratamiento (fueron ellos quienes llevaron al hospital a su hija y quienes cumplieron con la referencia al hospital de tercer nivel de atención). Los padres hicieron lo mejor por su hija, eso es innegable, pero el medio fue tan hostil que obstaculizó su deseo de que la paciente recuperara la salud.

La falta de apego a las indicaciones médicas ante una enfermedad amenazante para la vida constituyó una situación que ameritaba intervención urgente en el seno familiar, porque estuvo a nada de colapsar. No obstante, ¿por qué esperar a que la amenaza fuera de índole vital para intervenir? ¿No es la falta de educación de los menores un motivo para haber intervenido desde tiempo atrás?

El caso fue sometido al Comité de Bioética Hospitalaria con la finalidad de recibir una recomendación enfocada al mejor interés del menor y evaluar el grado y la magnitud de intervención que el hospital debería tener en el seno familiar. 
Como se discutió en los párrafos introductorios de este texto, cuando la adversidad o el daño al menor es mayor también será mayor la intervención que deberán tener terceros en su cuidado y atención, lo que quiere decir que no siempre se deberá interferir con la misma magnitud en la libertad que la familia tiene en el cuidado de sus integrantes, sino que dependerá de la gravedad del daño que se esté ocasionando al niño en el presente o hacia el futuro, con la finalidad de prevenir daños mayores.

Lo cierto es que la falta de apego a los cuidados, en una enfermedad que amenaza la vida, se considera de suma gravedad y el caso debe ser analizado hondamente, integrando todos los puntos de quiebre rescatables; por ello, en este caso se requirió el apoyo del Comité de Bioética Hospitalaria.

Para el Comité es preciso reconocer los criterios de capacidad de los niños para decidir sobre su atención médica cuando ellos mismos lo desean y lo pueden hacer; nada en nuestro análisis implica forzar al menor a decidir, ser presionado o alentado a decidir sobre su atención por sí mismo cuando no desea aún hacerlo. Un niño puede sentirse emocionalmente capaz de tomar decisiones, pero si en el ámbito de su salud no se siente con la facultad de asumir la responsabilidad de una decisión así, entonces, aunque sus demás capacidades están bien desarrolladas, podía ser dañino que asumiera esa responsabilidad. La paciente del caso es particular, pues no se encontraba en situación de abandono, orfandad ni situación de calle, y nuestro deber es procurar que los niños no sean «institucionalizados» y que puedan vivir en familia, ya sea propia o adoptada, en condiciones apropiadas y de bienestar, con la intervención de terceros cuando sea pertinente. Por lo anterior, cuando a los médicos tratantes se les interrogó sobre la opinión de la niña, expresaron que su voluntad era estar en casa con su familia; se planteó a la niña y sus hermanas la posibilidad de quedarse en otro sitio donde tuvieran «mejores» servicios (como comida, baño y cama) y que garantizara acudir a sus consultas y quimioterapias, pero ella y la familia fueron insistentes en quedarse en su casa, en compañía de sus hermanos. En una evaluación por parte del Departamento de Psicología se concluyó que la paciente poseía las capacidades para ser partícipe en las decisiones respecto a su tratamiento, pues tenía la facultad de comunicarse abiertamente, comprendía la información (planteada en términos que ella pudiera entender), entendía las repercusiones y consecuencias de no recibir su tratamiento (ya había vivido la muerte de ambos padres y comprendía que la leucemia le podía provocar la muerte) y podía dar razón y justificar el porqué de su decisión, así como plantear posibles soluciones realistas al problema planteado.

Se ha de notar que esta valoración fue particular y que hay una importantísima variación entre niños de la misma edad y sexo, por lo cual el Comité advirtió que se darían a la paciente los apoyos oportunos y pertinentes para garantizar la continuidad en su tratamiento y que, en caso de no lograr el objetivo, se tendría que intervenir jurídicamente para ponerla bajo la custodia y guardia del Estado. Mientras no fuera esta la situación, se vigilaría y apoyaría estrechamente por personal del hospital y del DIF.

El Comité de Bioética Hospitalaria es un órgano autónomo, interdisciplinario, plural y de carácter consultivo en el que se reflexiona, delibera y educa en un ambiente de libertad y tolerancia, y se analizan de forma sistemática los conflictos de valores y principios bioéticos que surgen durante el proceso de atención médica, pero su mera opinión no puede imponerse a la decisión médica. Por tanto, el Comité del HIMFG recomendó al médico tratante dar aviso al Departamento Jurídico de la Institución, así como continuar en contacto con el DIF a través de Trabajo Social del Departamento de Onco-hematología, que a su vez puso en operación las visitas domiciliarias semanales y el seguimiento de las citas por vía telefónica.

El Departamento Jurídico consideró que, ciertamente, la menor estaba en riesgo de muerte debido a la falta de cuidados, así como que estaba en el deseo de la familia otorgarlos; es indiscutible que los padres y las hermanas estaban a favor del mejor interés para la paciente, pero su situación social les impedía hacerlo correctamente.

Los tribunales reconocen que, actualmente, las alternativas para atender a estos pacientes por parte del Estado no son una garantía para su mejor bienestar.

El desarraigo familiar tiene consecuencias negativas para la maduración psicoemocional en la infancia y la adolescencia. La familia, con lo que se tenga de ella, sin lugar a duda es el mejor lugar para que un ser humano pueda nacer, crecer y madurar como persona sana en lo individual, y para ser una persona socialmente productiva. La legislación vigente en México dispone que, en el caso de que se encuentren familiares potencialmente disponibles para hacerse cargo del menor, no deberá retirarse la patria potestad, esperando que los integrantes de esa familia puedan responder como lo espera el bien común de la sociedad. La ley no describe en detalle criterios sobre las condiciones de vida que deben ofrecerse al niño, lo cual es comprensible por la heterogénea situación en la que 
pueden encontrarse los menores de nuestra sociedad. Se considera también que una gran mayoría de los menores en nuestro país vive en pobreza, que en ocasiones es extrema, y la solución más eficaz no es el retiro de la custodia, sino la intervención temprana de la sociedad en sí misma para fortalecer a las familias y acabar con el cáncer que es la pobreza y, como consecuencia directa, el abandono parcial o total de los menores.

El Grupo de Trabajo sobre Dilemas Éticos en Pediatría se integró en marzo de 2011, y desde entonces mantiene reuniones periódicas para lograr sus objetivos. El Grupo se compone de un equipo multidisciplinario (médicos, filósofos y académicos) e interinstitucional (HIMFG, Universidad Panamericana, Instituto Panamericano de Alta Dirección de Empresas, Hospital General de México Dr. Eduardo Liceaga, Universidad Anáhuac e Instituto Mexicano del Seguro Social). El propósito central del Grupo de Trabajo es contribuir a clarificar los dilemas éticos que se presentan en la práctica pediátrica cotidiana, así como dar atención a la búsqueda de un discernimiento ético apropiado en los casos representativos.

\section{Responsabilidades éticas}

Protección de personas y animales. Los autores declaran que para esta investigación no se han realizado experimentos en seres humanos ni en animales.

Confidencialidad de los datos. Los autores declaran que en este artículo no aparecen datos de pacientes.
Derecho a la privacidad y consentimiento informado. Los autores declaran que en este artículo no aparecen datos de pacientes.

\section{Conflicto de intereses}

Los autores declaran no tener ningún conflicto de intereses.

\section{Bibliografía}

1. Lewis C.G. A comparison of minors' and adults' pregnancy decisions. Am J Orthopsychiatry. 1980;50:446-453.

2. Melton GB, Koocher GP, Saks MJ. Children's competence to consent. US: Springer; 1983. p. 84.

3. Piaget J. El criterio moral en el niño. Barcelona: Fontanell; 1971.

4. Capron AM. The competence of children as self-deciders in biomediccal interventions. En: Gaylin W, Mcklin R, editores. Who speaks for the child. The problems os proxy consent. New York: Plenum Press; 1982. p. 94.

5. De Lora P. Autonomía personal, intervención médica y sujetos incapaces. Universidad Autónoma de Madrid. Enrahonar. 2008;40-41:123-40.

6. Gracia Guillen D. Sánchez-Jacobo M. Bioética y Pediatría Proyecto de Vida Plena. Madrid: Sociedad de Pediatría, Madrid y Castilla La Mancha. 2010; págs. 29-38.

7. UNICEF. Convención sobre los derechos del niño. Disponible en: https:// www.unicef.es/causas/derechos-ninos/convencion-derechos-ninos

8. Shoeman F. Parental discretion and children's rights: back-ground and implications for medical decision-making. J Med Philos. 1985;10:45-61.

9. Kemp P. La mundializacion de la ética. México: Distribuciones Fontamara; 2007.

10. Prilleltensky I, Sánchez-Valdés L, Rossiter A, Walsh-Bowers R. Applied ethics in mental health in Cuba: Part II. Power differentials, dilemmas, resources and limitations. Cuba: Lawrence Erlbaum Associates, Inc., Ethics \& Behavior. 2002;12:243-260.

11. Guadarrama-Orozco JH, Garduño EJ, Vargas LG, Viesca TC. Consentimiento informado y rechazo de los padres al tratamiento médico en edad pediátrica. El umbral de la tolerancia médica y social. Parte I. Bol Med Hosp Infant Mex. 2015;72:208-14.

12. Pinheiro PS. Informe del experto independiente para el estudio de la violencia contra los niños. Asamblea General de las Naciones Unidas. Nueva York: UNICEF; 2006. p. 19. 